\title{
Emergency reversal of antiplatelet agents in patients presenting with an intracranial hemorrhage: A clinical review
}

\author{
Peter G. Campbell, MD \\ Thomas Jefferson University \\ Anish Sen, BS \\ Jefferson Medical College \\ Sanjay Yadla, MD \\ Thomas Jefferson University \\ Pascal Jabbour, MD \\ Thomas Jefferson University \\ Jack Jallo, MD, PhD \\ Thomas Jefferson University \\ Follow this and additional works at: https://jdc.jefferson.edu/neurosurgeryfp \\ Part of the Medical Neurobiology Commons, and the Surgery Commons \\ Let us know how access to this document benefits you
}

\section{Recommended Citation}

Campbell, MD, Peter G.; Sen, BS, Anish; Yadla, MD, Sanjay; Jabbour, MD, Pascal; and Jallo, MD, $\mathrm{PhD}$, Jack, "Emergency reversal of antiplatelet agents in patients presenting with an intracranial hemorrhage: A clinical review" (2010). Department of Neurosurgery Faculty Papers. Paper 11. https://jdc.jefferson.edu/neurosurgeryfp/11

This Article is brought to you for free and open access by the Jefferson Digital Commons. The Jefferson Digital Commons is a service of Thomas Jefferson University's Center for Teaching and Learning (CTL). The Commons is a showcase for Jefferson books and journals, peer-reviewed scholarly publications, unique historical collections from the University archives, and teaching tools. The Jefferson Digital Commons allows researchers and interested readers anywhere in the world to learn about and keep up to date with Jefferson scholarship. This article has been accepted for inclusion in Department of Neurosurgery Faculty Papers by an authorized administrator of the Jefferson Digital Commons. For more information, please contact: JeffersonDigitalCommons@jefferson.edu. 
As submitted to:

\title{
World Neurosurgery
}

\section{And later published as:}

\section{"Emergency Reversal of Antiplatelet Agents in Patients Presenting with an Intracranial Hemorrhage: A Literature Review"}

\author{
Volume 74, Issue 2-3, August 2010, Pages 279-285 \\ DOI: 10.1016/j.wneu.2010.05.030
}

\author{
Peter G. Campbell, MD ${ }^{1}$ \\ Anish Sen, BS ${ }^{2}$ \\ Sanjay Yadla, $\mathrm{MD}^{1}$ \\ Pascal Jabbour, MD ${ }^{1}$ \\ Jack Jallo, MD, $\mathrm{PhD}^{1}$
}

Thomas Jefferson University

Department of Neurosurgery ${ }^{1}$

Jefferson Medical College ${ }^{2}$

Philadelphia, PA 
Corresponding Author:

Peter G. Campbell, MD

Department of Neurosurgery

909 Walnut St, $3^{\text {rd }}$ Floor

Philadelphia, PA 19107

Phone: (215) 503-7008

Email: peter.campbell@jeffersonhospital.org

\begin{abstract}
Objective: Prehospital use of antiplatelet agents has been associated with an increased risk for ICH as well as a secondary increase in ICH volume after the initial hemorrhage. Strategies to reestablish platelet aggregation are used in clinical practice, but without any established guidelines or recommendations. This article serves to evaluate the literature regarding "reversal" of antiplatelet agents in neurosurgical populations.
\end{abstract}

Methods: PUBMED and MEDLINE databases were searched for publications from 1966 to 2009 relating to intracranial hemorrhage and antiplatelet agents. The reference sections of recent articles, guidelines and reviews were reviewed and pertinent articles identified. Studies were classified by two broad subsets; those describing intracranial hemorrhage relatable to a traumatic mechanism and those with a spontaneous intracranial hemorrhage. Two independent auditors recorded and analyzed study design and the reported outcome measures.

Results: For the spontaneous intracranial hemorrhage group, 9 reports assessing antiplatelet effects on various outcome measures were identified. Eleven studies evaluating the use of prehospital antiplatlets prior to a traumatic intracranial hemorrhage were examined. 
Conclusion: The data assessing the relationship between outcome and prehospital antiplatelet agents in the setting of ICH is conflicting in both the trauma and the stroke literature. Only one retrospective review specifically addressed outcomes after attempted reversal with platelet transfusion. Further study is needed to determine whether platelet transfusion ameliorates hematoma enlargement and/or improves outcome in the setting of acute ICH.

Keywords: coagulopathy, antiplatelet agent, intracranial hemorrhage, aspirin, clopidogrel, trauma, stroke, platelet transfusion 


\section{Introduction}

Prophylactic treatment with anticoagulants and antiplatelet agents has clear benefits in select patients with cardiac and vascular diseases[1, 2]. Over the previous decade, there has been a significant increase in the use of these agents for primary prevention of stroke and heart disease and for prophylaxis and after percutaneous cardiac interventions. Patients experiencing intracranial hemorrhage (ICH) while fully anticoagulated have been reported to present with larger hematoma volumes and suffer a worse prognosis compared to control groups with normal coagulation profiles[3-5]. Further study has demonstrated that ICH progression in anticoagulated patients could be

halted, and mortality significantly reduced, with a prompt reversal of anticoagulation[6]. Some investigators have also reported that a prehospital antiplatelet regimen may be associated with hematoma expansion, an increased mortality rate, and a poor functional outcome[7-10]. As such, many institutions have established protocols to "reverse" the impairment of platelet aggregation caused by antiplatelet agents in patients presenting with ICH.

Several antiplatelet agents are commonly used in the outpatient setting. Aspirin is widely used for a variety of indications including risk reduction from transient ischemic 
attacks, prevention of primary myocardial infarction, and prevention of colorectal cancer[11]. Aspirin irreversibly binds to the cyclooxygenase-1 (COX-1) enzyme and subsequently inhibits production of thromboxane A2, a potent eicosanoid involved in platelet aggregation[12]. Non-steroidal anti-inflammatory drugs (NSAIDs) are typically used for relief from arthritis and headache pain. This class of medications acts upon on COX-1 in a reversible, dose-dependent inhibition; as the drug concentration decreases, COX-1 regains its enzymatic activity. Aspirin differs from NSAIDs functionally in that aspirin-induced COX-1 inhibition is irreversible, secondary to protein acetylation[13]. Clopidogrel and ticlopidine both selectively and irreversibly inhibit the $\mathrm{P} 2 \mathrm{Y}_{12}$ ADP receptor. These agents have been used effectively to treat a wide range of cardiovascular and cerebrovascular diseases, such as for prophylaxis after coronary or intracranial stent placement. Due to an active metabolite, clopidogrel in particular persists after cessation of the medication. Some studies have demonstrated a higher incidence of post-operative bleeding with clopidogrel as compared to aspirin, prompting some experts to recommend aggressive anti-platelet reversal for up to 4 days after the last known dose[14].

This review will focus on the treatment of ICH in patients taking prehospital NSAIDS, aspirin and clopidogrel (Plavix), and ticlopidine (Ticlid). Given the heterogeneity of the etiology of intracranial hemorrhages, spontaneous ICH and traumatic hemorrhages will be reviewed separately. The pertinent literature is evaluated and reviewed for evidence-based recommendations or guidelines to provide treatment guidance to clinicians for patients presenting with antiplatelet associated intracranial hemorrhages.

\section{Methods}




\section{Literature Review}

PUBMED and MEDLINE databases were searched for publications from 1966 to the present using MeSH terms 'antiplatelet', 'aspirin', 'clopidogrel (Plavix)', 'ticlopidine (Ticlid)', 'intracranial hemorrhage', 'head injury', 'traumatic hemorrhage', 'subdural hematoma', and 'stroke'. The search was limited to articles in the English language and those relating to human subjects over the age of 18 . Reference sections of recent articles, guidelines and reviews were reviewed and pertinent articles identified. Initially, articles identified as relevant were retrieved in abstract format. Full-text manuscripts were subsequently obtained for all original articles applicable to the current review.

Two authors independently evaluated all selected articles (PC, AS). For each study, study design (prospective or retrospective, use of control group, randomization), number of patients included and outcomes of the study were recorded. These results were used to assess if any evidence-based recommendations were able to be made from the literature.

\section{Spontaneous ICH}

Spontaneous intracerebral hemorrhage constitutes $10 \%$ to $15 \%$ of all strokes and is a devastating stroke subtype. Clinical trials of surgical interventions have failed to demonstrate a consistent benefit[15]. Many predictors of negative outcome have been consistently described in patients suffering from spontaneous $\mathrm{ICH}$, including oral anticoagulant therapy[3, 5]. Futhermore, warfarin use has been associated with larger volumes of ICH on baseline imaging, subsequent ICH growth, and increased mortality[16, 17]. Since hemorrhage expansion is an independent predictor of mortality and poor 
functional outcome, established guidelines require the prompt reversal of warfarininduced effects[18].

\section{Effects on Outcome in Spontaneous ICH}

Whether or not antiplatelet medication use at the time of spontaneous ICH influences hemorrhage expansion and neurologic outcome is controversial. Several studies with various designs, sizes, and exclusion criteria have displayed conflicting results (Table 1)[7, 9, 10, 19-23]. While these studies typically report the use of any antiplatelets together in one research arm, aspirin users account for the vast majority of the subpopulation in all studies. The efficacy of platelet transfusion as it relates to outcome was specifically assessed in only one study, but often not in the remainder, thus possibly confounding outcome comparison between the reports[24].

The placebo arms of several randomized studies have been evaluated to determine if any link exists between prehospital antiplatelet use and ICH volume. The Cerebral Hemorrhagic and NXY-059 Treatment trial reported no relationship between prior antiplatelet use and the volume of ICH on baseline $\mathrm{CT}$, ICH growth, or clinical outcome at 90 days[23]. In a phase II trial of rFVIIa for acute ICH, treatment with the recombinant factor and a longer time from onset to baseline $\mathrm{CT}$ were related to a decrease in hemorrhage growth in five models of ICH growth; four of the five models found no relationship between antiplatelet use and ICH growth[25]. In these studies, no transfusion of platelets was provided to study participants. Within these studies, at least $80 \%$ of the antiplatelet groups were taking ASA alone. The effect of antiplatelet use on outcomes in other prospective and retrospective analyses ranges from non-significant to a 
2.7-fold increased 30 day mortality rate and a 7.6-fold increase in hematoma enlargement[7, 9, 10, 19-23].

No study specifically addresses the effects of $\mathrm{P} 2 \mathrm{Y}_{12}$ ADP receptor inhibitors on spontaneous ICH. When a specific agent was specified by the study, clopidogrel and ticlopidine were being taken by 0 to $35.1 \%$ of the patients in the antiplatelet arms of studies in the literature[7, 9, 10, 19-23]. Toyoda et al.[10] reported on a series of 251 total patients with 57 antiplatelet users prior to spontaneous ICH. Ticlopidine was used secondary to the lack of availability of clopidogrel in Japan at the time. Twelve patients were on ticlopidine alone and 8 were taking a combination of ticlopidine and another antiplatelet (35.1\%). In this series, the use of prehospital antiplatelet agents was an independent predictor for hematoma enlargement $(\mathrm{p}<0.01)$, emergent evacuation $(\mathrm{p}=0.021)$ and emergent death[10].

\section{Reversal of Antiplatelet Agents in Spontaneous ICH}

In the setting of a spontaneous intracranial hemorrhage, attempts to "reverse" antiplatelet-induced effects upon platelets are the mainstay of treatment at most institutions[26]. However, based upon reports pertaining to mostly aspirin users with intracranial hemorrhage, some authors argue these actions are not clinically warranted[27]. While clopidogrel may have a safety profile similar to aspirin, several studies have demonstrated excessive bleeding in various clinical settings[28-31]. Furthermore, a randomized study reported the combination of clopidogrel and aspirin provided significantly more potent inhibition of platelet function when compared to monotherapy with aspirin[32]. To the authors' knowledge, no report exists dedicated to 
outcome comparison in the setting of spontaneous ICH in patients taking pre-hospital aspirin compared with those taking a $\mathrm{P} 2 \mathrm{Y}_{12}$ inhibitor. Given that clopidogrel-induced platelet dysfunction is typically more severe than aspirin; most neuro-intensivists and neurosurgeons continue recommend aggressive reversal of $\mathrm{P}_{2} \mathrm{Y}_{12}$ inhibitors in the setting of spontaneous ICH[26].

\section{Traumatic ICH}

Traumatic brain injury (TBI) is responsible for more than one million hospital visits, roughly 200,000 hospital admissions, and 50,000 deaths in the United States annually[33]. Given the considerable increase in the use of antiplatelet agents for stroke prevention and cardiac prophylaxis, many patients taking antiplatelet agents will subsequently experience intracranial hemorrhage secondary to TBI. In an attempt to reduce the increased morbidity and mortality associated with TBI in patients on antiplatelet or anticoagulant medications, some centers frequently reverse the effects of antiplatelet agents with platelet transfusion. In a study of anticoagulated patients with traumatic $\mathrm{ICH}$, rapid warfarin reversal led to a reduction in hemorrhage progression and a decrease in mortality $(\mathrm{p}<0.001)[6]$. However, the reversal of platelet dysfunction in patients with TBI on antiplatelet medications has not been fully investigated and no guidelines currently exist regarding this practice.

\section{Effects on Outcome in Traumatic ICH}

The current review includes 1 prospective and 10 retrospective studies that have evaluated the outcomes associated with prehospital antiplatelet use in patients admitted 
for traumatic head injuries (Table 2)[8, 34-43]. Of the retrospective evaluations, Rozzelle, et al.[35] compared 31 subdural hematoma patients on prehospital antiplatelet agents with controls and found no difference in-hospital mortality, but a 2.6-fold increase in craniotomies for this population. Mina, et al.[36] reported 34 patients with any TBI on preinjury warfarin, heparin, aspirin, NSAIDs, dipyridamole, clopidogrel, or pentoxifylline and found that this diverse group of patients had significantly more deaths when compared with controls not taking any anticoagulation or antiplatelet agents $(\mathrm{p}=0.006)$. In another retrospective evaluation, Ohm, et al.[38] examined 90 patients with traumatic ICH on prehospital antiplatelets in contrast to 89 matched controls not receiving antiplatelets. In-hospital mortality was $23 \%$ in the antiplatelet cohort compared with $8.9 \%$ in the control group $(\mathrm{p}=0.016)$ [38]. Ivascu, et al.[8] evaluated 109 patients on various antiplatelet agents as compared with 42 control patients and discovered those on preinjury antiplatelets were more likely to present with a higher grade hemorrhage than control patients not taking any antiplatelet agents $(\mathrm{p}=0.04)$. In another large retrospective review, 126 patients with hemorrhagic TBI were compared to controls not on any antiplatelet agent or anticoagulation. No significant differences between the groups were noted, leading the authors to conclude that preinjury antiplatelet or anticoagulant pharmacotherapy did not adversely impact outcome in elderly patients with blunt traumatic ICH[41].

Few studies have isolated the type of antiplatelet agent as it relates to outcome. In a prospective evaluation of the aspirin (ASA) effect upon traumatic brain injury, Spektor, et al.[37] reported 110 patients taking 100mg of ASA with mild or moderate head injury. However, traumatic intracranial hemorrhage was noted in only $27(24.5 \%)$ patients in the 
aspirin-treated group and in 31 patients $(25.6 \%)$ in the control group[37]. Thus, there was no statistically significant increase in the frequency or types of traumatic ICH in lowdose ASA users. Jones, et al.[39] attempted to evaluate the effects of clopidogrel in a general trauma population. Of the 41 patients isolated, only 25 had a head injury and only 9 had a traumatic ICH. While statistical reporting was lacking secondary to the small sample size, a higher percentage of patients in the clopidogrel group underwent cranial surgery, had rebleeding, and required transfusions of blood products than the control group, but mortality and hospital length of stay were not different than controls[39]. In an analysis of patients with traumatic ICH, Wong, et al.[40] reported on 131 patients taking preinjury ASA, clopidogrel and warfarin and found that clopidogrel demonstrated a 14.7-fold increase in the mortality after spontaneous ICH and resulted in a 3-fold increase in disposition to a long-term care facility. Though there are few studies that directly focus upon outcome associated with a specific antiplatelet agent, the two analyzing the use of clopidogrel prior to a traumatic ICH both reported worsened outcomes[39, 40].

\section{Reversal of Antiplatelets in Traumatic ICH}

Based on the majority of the studies available, it is conceivable that preinjury antiplatelet therapy may convert a minor traumatic ICH into a progressive $\mathrm{ICH}[11]$. However, currently available studies do not offer recommendations to clinicians on the use of reversal agents for patients with TBI on preinjury antiplatelet therapy. One study assessed patients who received platelet transfusions compared with those who did not to assess outcome differences in the setting of traumatic ICH[43]. In this multi-center 
retrospective series, 166 patients with traumatic $\mathrm{ICH}$ received platelet transfusions, and 162 did not. The mortality rate for the transfused patients was $17.5 \%$, while mortality for those who did not receive platelet transfusions was $16.7 \%(\mathrm{p}=0.85)[43]$. Unfortunately, statistical analysis was not reported for any specific anti-platelet agent. Hence, though at first glance it may seem that, as with warfarin, reversal of antiplatelet effects with platelet transfusion should lead to a reduction in mortality and morbidity in the setting of a traumatic $\mathrm{ICH}$, the only study that assessed this hypothesis did not find evidence to support this practice.

\section{Options for Reversal}

The literature regarding "reversal" of prehospital aspirin therapy after acute intracranial hemorrhage is equivocal. At many institutions, it is standard practice to recommend platelet transfusion in the setting of intracranial hemorrhage in an effort to stem the salicylate effect upon platelet aggregation[44]. Although there is no specific antidote, the aspirin effect is believed to be reversed by one platelet transfusion (1 single donor or 5 pooled concentrates)[26, 45]. Furthermore, other agents for reversal of the aspirin effect have received basic investigation. Desmopressin seems capable of correcting aspirin-induced platelet dysfunction, although large clinical studies describing relevant outcome parameters have not been undertaken[46-48]. Altman, et al.[49] treated healthy volunteers with aspirin and found that recombinant factor VIIa (rFVIIIa) combined with other agonists was able to reverse the anti-aggregating effect of aspirin. No randomized study has been reported outcomes of acute $\mathrm{ICH}$ with reference to the reversal the aspirin effect. 
Clopidogrel belongs to the class of thienopyridine derivatives, which act by blocking the ADP receptor on the platelet. Like aspirin, no specific antidote exists for the reversal of these agents. In an ex vivo study on healthy volunteers given aspirin and clopidogrel, a platelet transfusion consisting of 10 to 12.5 concentrate units successfully restored platelet reactivity[45]. However, given that an active metabolite of clopidogrel persists after cessation of the medication, some experts recommend continued platelet transfusion 4 or 5 days after the last dose of the medication[44]. In another ex vivo study of healthy volunteers treated with aspirin plus clopidogrel, rFVIIa reversed the inhibitory platelet effects of these agents[49]. While the clinical utility of desmopressin has not been assessed in the ICH population, Ranucci, et al.[50] reported the antiplatelet effects of clopidogrel and aspirin were partially reversed by an intravenous infusion of desmopressin after carotid endarterectomy. No randomized studies have been reported to assess outcomes after the reversal of thienopyridines in the setting of acute ICH.

\section{Proposed Protocol for Reversal of Antiplatelets}

Given the various clinical reports currently available and the absence of any established guidelines in the literature, the current authors' have developed a multidisciplinary institutional protocol for antiplatelet reversal in the setting of an acute ICH (Figure 1). The authors would like to accentuate the fact that this protocol is based purely upon the recommendations of a local multi-disciplinary team comprised of hematologists, intensivists and neurosurgeons. Patients presenting with an intracranial hemorrhage on ASA alone are given 5 platelet concentrate units upon admission. Those patients presenting with an acute ICH on clopidogrel with small hemorrhages and a neurologic exam that is able to be followed receive an initial transfusion of 10 platelet 
concentrate units. For those patients with a severe acute ICH on clopidogrel, 10 units of platelets are transfused initially with $0.3 \mu \mathrm{g} / \mathrm{kg}$ of desmopressin, and platelets are subsequently transfused every 12 hours for the next 48 hours. Data regarding clinical outcomes is currently being accumulated.

\section{Discussion}

\section{Effect of pharmacokinetics}

There is a known substantial variation in patient response to antiplatelet medications[51]. This diversification of molecular effectiveness amongst patients may, in part, explain some of the conflicting outcome results seen in the retrospective reviews presented in Table 1 and Table 2. Recently, Naidech et al.[52-54] reported several studies linking objective measures of platelet function with increased mortality, increased early clot growth, worse functional outcomes at 3 months and intraventricular extension after ICH. These authors used two bedside optical detection systems which measured platelet induced aggregation inhibition due to aspirin and clopidogrel. They determined that abnormal platelet activity results, not the reported use of antiplatelet medications were associated with these negative outcomes and posited that increasing platelet activity may be a more appropriate potential target to improve outcomes after ICH[53]. While more studies at various centers are needed to confirm these findings, these studies provide early evidence that restoring the platelet activity in specifically targeted patients directly upon admission may be of benefit. 
Potent metabolites of the thienopyridines (clopidogrel and ticlopidine) irreversibly inhibit the $\mathrm{P} 2 \mathrm{Y}_{12}$ receptor. Thus, urgent surgical intervention in patients taking these agents is often problematic. From the cardiac literature, clopidogrel treatment within 4-5 days of a coronary artery bypass grafting procedures has been associated with increased blood loss, reoperation for bleeding, increased transfusion requirements, and prolonged intensive care unit and hospital stays[14, 29, 55]. As emergent surgery is often a necessity in the setting of acute coronary syndrome, two direct and reversible $\mathrm{P} 2 \mathrm{Y}_{12}$ antagonists with rapid onset of action have been developed. These new agents, which are currently in Phase III trials, are attractive alternatives to the thienopyridines, especially in situations whereby the rapid inhibition of platelet aggregation or its quick reversal are required[56]. Thus, with the addition of these new $\mathrm{P}_{2} \mathrm{Y}_{12}$ inhibitors, physicians will be able to prescribe a more patient-specific antiplatelet regimen based upon individual riskbenefit analysis.

\section{Summary of Findings}

The data assessing the relationship between outcomes and prehospital antiplatelet agents in the setting of ICH is contradictory in both the trauma and the stroke literature. The majority of studies reveal a worsened outcome in ICH after taking ASA. After clopidogrel ingestion both dedicated studies in the trauma literature noted worse outcomes, while the study including the highest percentage of patients taking thienopyridines in the spontaneous ICH literature noted worse outcomes as well when compared to controls[10, 39, 40]. Only one retrospective review assessed outcomes after platelet transfusion, the mainstay of antiplatelet reversal at many institutions. Currently, 
a randomized trial is recruiting patients in order to determine whether platelet transfusion ameliorates hematoma enlargement in the setting of acute ICH[7]. At present, the literature contains insufficient information to establish any guidelines or treatment recommendations. In light of this, the current authors have proposed a protocol for antiplatelet reversal in both spontaneous and traumatic acute ICH.

\section{References}

1. Hirsh, J. and R. Raschke, Heparin and low-molecular-weight heparin: the Seventh ACCP Conference on Antithrombotic and Thrombolytic Therapy. Chest, 2004. 126(3 Suppl): p. 188S-203S.

2. Patrono, C., et al., Platelet-active drugs: the relationships among dose, effectiveness, and side effects: the Seventh ACCP Conference on Antithrombotic and Thrombolytic Therapy. Chest, 2004. 126(3 Suppl): p. 234S-264S.

3. Flibotte, J.J., et al., Warfarin, hematoma expansion, and outcome of intracerebral hemorrhage. Neurology, 2004. 63(6): p. 1059-64.

4. Franke, C.L., et al., Intracerebral hematomas during anticoagulant treatment.[see comment]. Stroke, 1990. 21(5): p. 726-30.

5. Rosand, J., et al., The effect of warfarin and intensity of anticoagulation on outcome of intracerebral hemorrhage.[see comment]. Archives of Internal Medicine, 2004. 164(8): p. 880-4.

6. Ivascu, F.A., et al., Rapid warfarin reversal in anticoagulated patients with traumatic intracranial hemorrhage reduces hemorrhage progression and 
mortality. Journal of Trauma-Injury Infection \& Critical Care, 2005. 59(5): p. 1131-7; discussion 1137-9.

7. Saloheimo, P., et al., Regular aspirin-use preceding the onset of primary intracerebral hemorrhage is an independent predictor for death.[see comment]. Stroke, 2006. 37(1): p. 129-33.

8. Ivascu, F.A., et al., Predictors of mortality in trauma patients with intracranial hemorrhage on preinjury aspirin or clopidogrel. Journal of Trauma-Injury Infection \& Critical Care, 2008. 65(4): p. 785-8.

9. Roquer, J., et al., Previous antiplatelet therapy is an independent predictor of 30day mortality after spontaneous supratentorial intracerebral hemorrhage. Journal of Neurology, 2005. 252(4): p. 412-6.

10. Toyoda, K., et al., Antiplatelet therapy contributes to acute deterioration of intracerebral hemorrhage.[see comment]. Neurology, 2005. 65(7): p. 1000-4.

11. McMillian, W.D. and F.B. Rogers, Management of prehospital antiplatelet and anticoagulant therapy in traumatic head injury: a review. Journal of TraumaInjury Infection \& Critical Care, 2009. 66(3): p. 942-50.

12. Kalgutkar, A.S., et al., Aspirin-like molecules that covalently inactivate cyclooxygenase-2.[see comment]. Science, 1998. 280(5367): p. 1268-70.

13. Vane, J.R., Y.S. Bakhle, and R.M. Botting, Cyclooxygenases 1 and 2. Annual Review of Pharmacology \& Toxicology, 1998. 38: p. 97-120.

14. Yende, S. and R.G. Wunderink, Effect of clopidogrel on bleeding after coronary artery bypass surgery. Critical Care Medicine, 2001. 29(12): p. 2271-5. 
15. Mendelow, A.D., et al., Early surgery versus initial conservative treatment in patients with spontaneous supratentorial intracerebral haematomas in the International Surgical Trial in Intracerebral Haemorrhage (STICH): a randomised trial.[see comment]. Lancet, 2005. 365(9457): p. 387-97.

16. Cucchiara, B., et al., Hematoma growth in oral anticoagulant related intracerebral hemorrhage. Stroke, 2008. 39(11): p. 2993-6.

17. Flaherty, M.L., et al., Warfarin use leads to larger intracerebral hematomas. Neurology, 2008. 71(14): p. 1084-9.

18. Broderick, J., et al., Guidelines for the management of spontaneous intracerebral hemorrhage in adults: 2007 update: a guideline from the American Heart Association/American Stroke Association Stroke Council, High Blood Pressure Research Council, and the Quality of Care and Outcomes in Research Interdisciplinary Working Group.[see comment][reprint in Circulation. 2007 Oct 16;116(16):e391-413; PMID: 17938297]. Stroke, 2007. 38(6): p. 2001-23.

19. Cantalapiedra, A., et al., Oral anticoagulant treatment: risk factors involved in 500 intracranial hemorrhages. Journal of Thrombosis \& Thrombolysis, 2006. 22(2): p. 113-20.

20. Foerch, C., et al., Pretreatment with antiplatelet agents is not independently associated with unfavorable outcome in intracerebral hemorrhage.[see comment]. Stroke, 2006. 37(8): p. 2165-7.

21. Caso, V., et al., Effect of on-admission antiplatelet treatment on patients with cerebral hemorrhage. Cerebrovascular Diseases, 2007. 24(2-3): p. 215-8. 
22. Lacut, K., et al., Antiplatelet drugs and risk of venous thromboembolism: results from the EDITH case-control study. Haematologica, 2008. 93(7): p. 1117-8.

23. Sansing, L.H., et al., Prior antiplatelet use does not affect hemorrhage growth or outcome after ICH.[see comment]. Neurology, 2009. 72(16): p. 1397-402.

24. Creutzfeldt, C.J., et al., Prior antiplatelet therapy, platelet infusion therapy, and outcome after intracerebral hemorrhage. Journal of Stroke \& Cerebrovascular Diseases, 2009. 18(3): p. 221-8.

25. Mayer, S.A., et al., Safety and feasibility of recombinant factor VIIa for acute intracerebral hemorrhage. Stroke, 2005. 36(1): p. 74-9.

26. Beshay JE, M.H., Madden C, Yu W, Sarode R., Emergency reversal of anticoagulation and antiplatelet therapies in neurosurgical patients. Journal of Neurosurgery, 2009. [Epub ahead of print].

27. Broderick, J.P., Evidence against rapid reversal of antiplatelet medications in acute intracerebral hemorrhage.[comment]. Neurology, 2009. 72(16): p. 1376-7.

28. Berger, J.S., et al., Impact of clopidogrel in patients with acute coronary syndromes requiring coronary artery bypass surgery: a multicenter analysis. $\mathrm{J}$ Am Coll Cardiol, 2008. 52(21): p. 1693-701.

29. Hongo, R.H., et al., The effect of clopidogrel in combination with aspirin when given before coronary artery bypass grafting. J Am Coll Cardiol, 2002. 40(2): p. $231-7$.

30. Kapetanakis, E.I., et al., Clopidogrel administration prior to coronary artery bypass grafting surgery: the cardiologist's panacea or the surgeon's headache? Eur Heart J, 2005. 26(6): p. 576-83. 
31. Kim, J.H., et al., Clopidogrel use and bleeding after coronary artery bypass graft surgery. Am Heart J, 2008. 156(5): p. 886-92.

32. Serebruany, V.L., et al., Effects of clopidogrel and aspirin in combination versus aspirin alone on platelet activation and major receptor expression in patients after recent ischemic stroke: for the Plavix Use for Treatment of Stroke (PLUTOStroke) trial. Stroke, 2005. 36(10): p. 2289-92.

33. Thurman, D. and J. Guerrero, Trends in hospitalization associated with traumatic brain injury. JAMA, 1999. 282(10): p. 954-7.

34. Reymond, M.A., et al., Aspirin as a risk factor for hemorrhage in patients with head injuries. Neurosurg Rev, 1992. 15(1): p. 21-5.

35. Rozzelle, C.J., J.L. Wofford, and C.L. Branch, Predictors of hospital mortality in older patients with subdural hematoma. J Am Geriatr Soc, 1995. 43(3): p. 240-4.

36. Mina, A.A., et al., Intracranial complications of preinjury anticoagulation in trauma patients with head injury. J Trauma, 2002. 53(4): p. 668-72.

37. Spektor, S., et al., Low-dose aspirin prophylaxis and risk of intracranial hemorrhage in patients older than 60 years of age with mild or moderate head injury: a prospective study. J Neurosurg, 2003. 99(4): p. 661-5.

38. Ohm, C., et al., Effects of antiplatelet agents on outcomes for elderly patients with traumatic intracranial hemorrhage. J Trauma, 2005. 58(3): p. 518-22.

39. Jones, K., et al., The effects of preinjury clopidogrel use on older trauma patients with head injuries. Am J Surg, 2006. 192(6): p. 743-5.

40. Wong, D.K., F. Lurie, and L.L. Wong, The effects of clopidogrel on elderly traumatic brain injured patients. J Trauma, 2008. 65(6): p. 1303-8. 
41. Fortuna, G.R., et al., The impact of preinjury antiplatelet and anticoagulant pharmacotherapy on outcomes in elderly patients with hemorrhagic brain injury. Surgery, 2008. 144(4): p. 598-603; discussion 603-5.

42. Ahmed, N., et al., Impact of preinjury anticoagulation in patients with traumatic brain injury. South Med J, 2009. 102(5): p. 476-80.

43. Downey, D.M., et al., Does platelet administration affect mortality in elderly head-injured patients taking antiplatelet medications? Am Surg, 2009. 75(11): p. 1100-3.

44. Herman, J., Benson K, ed. Platelet Transfusion Therapy. Transfusion Therapy: Clinical Principles and Practice, second edition, ed. M. PD. 2004, AABB Press: Bethesda, MD. 335-353.

45. Vilahur, G., et al., Normalization of platelet reactivity in clopidogrel-treated subjects. J Thromb Haemost, 2007. 5(1): p. 82-90.

46. Flordal, P.A. and S. Sahlin, Use of desmopressin to prevent bleeding complications in patients treated with aspirin. Br J Surg, 1993. 80(6): p. 723-4.

47. Gratz, I., et al., The effect of desmopressin acetate on postoperative hemorrhage in patients receiving aspirin therapy before coronary artery bypass operations. $\mathrm{J}$ Thorac Cardiovasc Surg, 1992. 104(5): p. 1417-22.

48. Levi, M., Emergency reversal of antithrombotic treatment. Intern Emerg Med, 2009. 4(2): p. 137-45.

49. Altman, R., et al., Recombinant factor VIIa reverses the inhibitory effect of aspirin or aspirin plus clopidogrel on in vitro thrombin generation. J Thromb Haemost, 2006. 4(9): p. 2022-7. 
50. Ranucci, M., et al., Platelet mapping and desmopressin reversal of platelet inhibition during emergency carotid endarterectomy. J Cardiothorac Vasc Anesth, 2007. 21(6): p. 851-4.

51. Helgason, C.M., et al., Aspirin response and failure in cerebral infarction. Stroke, 1993. 24(3): p. 345-50.

52. Naidech, A.M., et al., Reduced platelet activity is associated with more intraventricular hemorrhage. Neurosurgery, 2009. 65(4): p. 684-8; discussion 688.

53. Naidech, A.M., et al., Platelet activity and outcome after intracerebral hemorrhage. Ann Neurol, 2009. 65(3): p. 352-6.

54. Naidech, A.M., et al., Reduced platelet activity is associated with early clot growth and worse 3-month outcome after intracerebral hemorrhage. Stroke, 2009. 40(7): p. 2398-401.

55. Chen, L., et al., Clopidogrel and bleeding in patients undergoing elective coronary artery bypass grafting. J Thorac Cardiovasc Surg, 2004. 128(3): p. 42531.

56. Cattaneo, M., New P2Y12 blockers. J Thromb Haemost, 2009. 7 Suppl 1: p. 2625. 
Table 1: Literature review of the effect of antiplatelet agents upon outcome in spontaneous ICH 
Table 2: Literature review of the effect of antiplatelet agents upon outcome in the setting of traumatic ICH. 
Figure 1: Proposed protocol for reversal of antiplatelet agents in the setting of acute intracranial hemorrhage. 
